\title{
Tumor response from durvalumab (MEDI4736) + tremelimumab treatment in patients with advanced non-small cell lung cancer (NSCLC) is observed regardless of PD-L1 status
}

\author{
Naiyer Rizvi ${ }^{1 *}$, Jamie Chaft ${ }^{2}$, Ani Balmanoukian ${ }^{3}$, Sarah B Goldberg ${ }^{4}$, Rachel E Sanborn ${ }^{5}$, Keith E Steele ${ }^{6}$, \\ Marlon C Rebelatto ${ }^{6}$, Yu Gu ${ }^{6}$, Joyson J Karakunnel ${ }^{6}$, Scott Antonia ${ }^{7}$
}

From 30th Annual Meeting and Associated Programs of the Society for Immunotherapy of Cancer (SITC 2015) National Harbor, MD, USA. 4-8 November 2015

\section{Background}

As single agents, durvalumab (MEDI4736), a human IgG1 anti-PD-L1 antibody, and tremelimumab, a human IgG2 anti-CTLA-4 antibody, have shown acceptable safety profiles and antitumor activity. Similar to other anti-PD-L1/anti-PD-1 monotherapies, durvalumab has shown greater objective tumor response rates in PD-L1positive patients compared with PD-L1-negative patients. Anti-CTLA4 therapies activate T-cells and may increase immune infiltrate and PD-L1 expression in tumor cells and tumor infiltrating immune cells. Thus, combination therapy with durvalumab and tremelimumab could be active in NSCLC regardless of baseline PD-L1 expression.

\section{Methods}

This is a phase 1, open-label, dose-escalation/expansion study (NCT02000947) of D+T in patients with Stage III/ IV NSCLC (any number of prior lines of therapy; immunotherapy-naïve). The primary endpoint is safety and tolerability; secondary endpoints include investigatorreported RECIST v1.1 response. PD-L1 expression was tested retrospectively using an immunohistochemical assay (Ventana).

\section{Results}

As of 1 June 2015, 102 patients received treatment in the dose escalation phase; combinations of durvalumab [3 $\mathrm{mg} /$ $\mathrm{kg}$ (D3) to $20 \mathrm{mg} / \mathrm{kg}$ (D20) every 2 (q2w) or 4 weeks

${ }^{1}$ Columbia University Medical Center, New York, NY, USA

Full list of author information is available at the end of the article (q4w)] and tremelimumab [1 mg/kg (T1) to $3 \mathrm{mg} / \mathrm{kg}$ (T3)] $\mathrm{q} 4 \mathrm{w}$, plus a D15 + T10 combination, were explored. Across all cohorts, $80 \%$ and $42 \%$ of patients had $\geq 1$ treatment-related AE (any Grade and Grade 3/4, respectively); $28 \%$ discontinued treatment due to a related AE. A greater frequency of AEs, without a corresponding increase in tumor response, was seen with increasing $\mathrm{T}$ dose. In the combined T1 cohort (D10-D20), 73\% and 30\% of patients had $\geq 1$ related AE (any Grade and Grade 3/4, respectively); $16 \%$ discontinued treatment due to a related AE. There were 3 treatment-related deaths (myasthenia gravis, T1; pericardial effusion, T1; neuromuscular disorder, T3).

84 patients (73 EGFR/ALK wild-type; 77 non-squamous; 48 with $\geq 2$ prior lines of therapy) were evaluable for response (Table 1). The overall response rate (confirmed+unconfirmed) was $25 \%$. Higher response rates were observed in those with 1 vs $\geq 2$ prior therapies. Response rates do not appear dependent on PD-L1 status: 35\% (PD-L1-positive), 22\% (PD-L1-negative, $<25 \%$ tumor cell staining) and 33\% (PD-L1-negative, 0\% tumor cell staining). Similar findings were observed for the combined $\mathrm{T} 1$ cohort. $\mathrm{D}+\mathrm{T}$ also showed good durability of response similar to that seen for monotherapy.

\section{Conclusions}

$\mathrm{D}+\mathrm{T}$ at selected phase 3 dose (D20, T1) has a manageable tolerability profile and anti-tumor activity in NSCLC. Unlike anti- PD-1/PD-L1 monotherapies, the combination of $\mathrm{D}+\mathrm{T}$ appears to be active regardless of PD-L1 status, including even in patients with no tumor cell membrane PD-L1 staining, a setting where patients would not be expected to derive significant benefit from 
Table 1 Response rates (Confirmed/unconfirmed with $\geq 16$ weeks follow-up)

\begin{tabular}{|c|c|c|c|c|c|c|c|c|}
\hline & \multicolumn{4}{|c|}{ Overall population } & \multicolumn{4}{|c|}{ EGFR/ALK wild-type population } \\
\hline & \multicolumn{2}{|c|}{ All cohorts } & \multicolumn{2}{|c|}{ Combined cohort: D10-20 q4w or q2w + T1 } & \multicolumn{2}{|c|}{ All cohorts } & \multicolumn{2}{|c|}{ Combined cohort: D10-20 q4w or q2w + T } \\
\hline & $\mathrm{n} / \mathrm{N}(\%)$ & $95 \% \mathrm{Cl}$ & $\mathrm{n} / \mathrm{N}(\%)$ & $95 \% \mathrm{Cl}$ & $\mathrm{n} / \mathrm{N}(\%)$ & $95 \% \mathrm{Cl}$ & $\mathrm{n} / \mathrm{N}(\%)$ & $95 \% \mathrm{Cl}$ \\
\hline All patients & $21 / 84(25)$ & $16-36$ & $11 / 39(28)$ & $15-45$ & $21 / 73(29)$ & $19-41$ & $11 / 34(32)$ & $17-51$ \\
\hline $\mathrm{PD}^{-\mathrm{L}^{+}}{ }^{+}(\geq 25 \%)$ & $7 / 20(35)$ & $15-59$ & $3 / 9(33)$ & $8-70$ & $7 / 17(41)$ & $18-67$ & 3/9 (33) & $8-70$ \\
\hline PD-L1' $^{-}(<25 \%)$ & $11 / 49(22)$ & $12-37$ & $6 / 23(26)$ & $10-48$ & $11 / 45(24)$ & $13-40$ & $6 / 19(32)$ & $13-57$ \\
\hline PD-L1'- (0\%) & 9/27 (33) & $17-54$ & $6 / 12(50)$ & $21-79$ & $9 / 26(35)$ & $17-56$ & 6/11 (55) & $23-83$ \\
\hline All $2 \mathrm{~L}$ patients & $15 / 32(47)$ & $29-65$ & $7 / 16$ (44) & $20-70$ & $15 / 31(48)$ & $30-67$ & $7 / 15$ (47) & $21-73$ \\
\hline $\mathrm{PD}^{-\mathrm{L1}^{+}}(\geq 25 \%)$ & $6 / 8(75)$ & $35-97$ & $2 / 3(67)$ & 9-99 & $6 / 8(75)$ & $35-97$ & $2 / 3(67)$ & $9-99$ \\
\hline PD-L1' $(<25 \%)$ & 7/18 (39) & $17-64$ & $4 / 11(36)$ & $11-69$ & 7/17 (41) & $18-67$ & $4 / 10(40)$ & $12-74$ \\
\hline PD-L1 ${ }^{-}(0 \%)$ & $6 / 8(75)$ & $35-97$ & $4 / 5(80)$ & $28-100$ & $6 / 8(75)$ & $35-97$ & $4 / 5(80)$ & $28-100$ \\
\hline
\end{tabular}

$2 \mathrm{~L}$, second line: 1 prior line of therapy, receiving $\mathrm{D}+\mathrm{T}$ in second line

anti-PD-1/PD-L1 monotherapy over current standard of care $[1,2]$.

\section{Authors' details}

${ }^{1}$ Columbia University Medical Center, New York, NY, USA. ${ }^{2}$ Memorial Sloan Kettering Cancer Center, New York, NY, USA. ${ }^{3}$ The Angeles Clinic and Research Institute, Los Angeles, CA, USA. ${ }^{4}$ Yale University, Yale Cancer Center, New Haven, CT, USA. ${ }^{5}$ Earle A. Chiles Research Institute, Providence Cancer Center, Portland, OR, USA. ${ }^{6}$ Medlmmune, Gaithersburg, MD, USA. ${ }^{7}$ Moffitt Cancer Center, Tampa, FL, USA.

Published: 4 November 2015

\section{References}

1. Paz-Ares L, et al: ASCO 2015, abstract LBA109.

2. Vansteenkiste J, et al: ECC 2015, abstract 14LBA.

doi:10.1186/2051-1426-3-S2-P193
Cite this article as: Rizvi et al.: Tumor response from durvalumab
(MEDI4736) + tremelimumab treatment in patients with advanced non-
small cell lung cancer (NSCLC) is observed regardless of PD-L1 status.
Journal for ImmunoTherapy of Cancer 2015 3(Suppl 2):P193.

Submit your next manuscript to BioMed Central and take full advantage of:

- Convenient online submission

- Thorough peer review

- No space constraints or color figure charges

- Immediate publication on acceptance

- Inclusion in PubMed, CAS, Scopus and Google Scholar

- Research which is freely available for redistribution

Submit your manuscript at www.biomedcentral.com/submit
C Biomed Central 